No. 562

Februar 2017

Two-phase Dusty Fluid Flow Along a Rotating Axi-symmetric Round-Nosed Body

S. Siddiqa, N. Begum,

M. A. Hossain, R. S. R. Gorla 


\title{
Two-phase Dusty Fluid Flow Along a Rotating Axi-symmetric Round-Nosed Body
}

\author{
Sadia Siddiqa*,1, Naheed Begum*, ${ }^{*}$, M. A. Hossain ${ }^{\S}$, Rama Subba Reddy Gorla ${ }^{\ddagger}$ \\ ${ }^{*}$ Department of Mathematics, COMSATS Institute of Information Technology, Kamra \\ Road, Attock, Pakistan \\ ${ }^{\dagger}$ Institute of Applied Mathematics (LSIII), TU Dortmund, Vogelpothsweg 87, D-44221 \\ Dortmund, Germany \\ $\S$ Department of Mathematics, University of Dhaka, Dhaka, Bangladesh \\ ${ }^{\ddagger}$ Department of Mechanical $\&$ Civil Engineering, Purdue University Northwest, Westville, \\ IN 46391
}

\begin{abstract}
This article is concerned with the class of solutions of gas boundary layer containing uniform, spherical solid particles over the surface of rotating axi-symmetric roundnosed body. By using the method of transformed coordinates, the boundary-layer equations for two-phase flow are mapped into a regular and stationary computational domain and then solved numerically by using implicit finite difference method. In this study, a rotating hemisphere is used as a particular example to elucidate the heat transfer mechanism near the surfaces of round nosed bodies. We will also investigate whether the presence of dust particles in carrier fluid disturb the flow characteristics associated with rotating hemisphere or not. A comprehensive parametric analysis is presented to show the influence of the particle loading, the buoyancy ratio parameter and the surface of rotating hemisphere on the numerical findings. In the absence of dust particles, the results are graphically compared with existing data in the open literature and an excellent agreement has been found. It is noted that, the concentration of dust particles parameter, $D_{\rho}$, strongly influence the heat transport rate near the leading edge.
\end{abstract}

Keywords: Natural Convection, Two-phase, Dusty Fluid, Round-Nosed Body.

\section{Introduction}

The class of flows along axi-symmetric round nosed bodies has received a notable attention due to their frequent occurrence in shape and optimization of the flying vehicles. For instance, the round-nosed shapes can be visualized on subsonic vehicles. All commercial aircrafts that fly at subsonic speed (i.e. less than the speed of sound) usually have the ideal shape as rounded (parabolic) nose. The interesting effect of nose blunting has been found to be a drag reduction, for some levels of rounding radius, compared with the corresponding sharp nosed body flow. Moreover, rotating spheres and cones are also used as nose cones in aeroengine and spinning projectile applications. The non-similar boundary layer analysis is accepted to figure out the distribution of skin friction and heat transfer coefficients along the wall. Rotating round-nosed bodies have been initially exploited by Suwono [1] to report the effects of buoyancy forces on laminar boundary layer flow. In this paper, the author presented the theoretical analysis of a steady flow and heat transfer for the case of rotating hemispheres for Prandtl number $(0.72<\mathrm{P} r<100)$ and buoyancy ratio parameter

\footnotetext{
${ }^{1}$ Corresponding Author.

Email: saadiasiddiqa@gmail.com
} 
$(0 \leq \lambda<\infty)$ and concluded that the buoyancy forces are more pronounced for flows engendered by rotating bodies rather than the flows over submerged bodies. Based on the analysis of [1], the studies of boundary layer flow near rotating axi-symmetric round nosed bodies were presented by Hossain et al. ([2]-[3]). In these references, the authors presented the numerical solutions by using the Keller-box method as well as local non-similarity method for the application of rotating hemispheres. Recently, Siddiqa et al. [4] reported the influence of temperature-dependent density on natural convection flow near rotating axi-symmetric round-nosed body. In this paper, the authors performed the numerical simulations for the case of hemisphere which is rotating in an infinite motionless medium and reported that the transverse curvature of the surface has a significant influence on flow characteristics. In all above-mentioned studies, attention has been given to viscous fluid, which is free from all impurities (clear fluid). But, pure fluid is rarely available in many practical situations, for instance, common fluids like air and water contain impurities like dust particles. Therefore, the class of fluids having more than one phase has attracted the attention of several investigators due to their wide range of applications in many technical areas like fluidization, flow in rocket tubes, combustion, solid rocket exhaust nozzles, nuclear reactors with gas-solid feeds, ablation cooling, blast waves moving over the earths surface, conveying of powdered materials, environmental pollutants, petroleum industry, purification of crude oil, physiological flows and many other fields (see [5]). In this regard, Farbar and Morley [6] were the first to analyze the gas-particulate suspension on experimental grounds. After that, Marble [7] studied the problem of dynamics of a gas containing small solid particles and developed the equations for gas-particle flow systems. Singleton [8] was the first to study the boundary layer analysis for dusty fluid and later on several attempts were made to conclude the physical insight of such two-phase flows (see Ref. [9]-[17]) under different physical circumstances. Very recently, numerical solutions for the influence of thermal radiation on natural convection flow of dusty fluid past a vertical wavy frustum of a cone are presented by Siddiqa et al. [18]. In this paper, the authors solved the coupled system of equations numerically through implicit finite difference method for two important particulate suspension, namely, water-metal mixture and air-metal mixture.

However, the interaction of two-phase dusty fluid along an axi-symmetric roundnosed bodies is not considered so far in the literature. Due to the less emphasis given to blunt bodies, present study is conducted in which natural convection flow of two-phase dusty fluid is modeled along an axi-symmetric round-nosed body. The governing boundary equations are reduced to convenient form by the introduction of primitive variable formulation and then the resulting system of parabolic partial differential equations are solved numerically by implicit finite difference method. Numerical results are obtained with a view to disclose the quantitative response of various flow parameters on the components of carrier phase (or particle phase) velocity, carrier phase (or particle phase) temperature, wall shear stress and wall heat transfer rate.

\section{Mathematical Formulation}

Consider a laminar boundary layer flow of a mixture of solid spherical particles homogeneously distributed in a continuous carrier fluid or gas along a rotating round-nosed body of revolution. The flow is due to the combined forces of thermal buoyancy and the angular rotation of the axi-symmetric body. Following assumption have been made for the problem 
under consideration:

- The $x$-coordinate is measured from the stagnation point along the surface, $y$-coordinate is taken normal to the surface and $z$-coordinate is in the direction of the rotation as depicted in Fig. 1.

- The round nosed body is moving with the constant angular velocity, $\Omega$, and the surface of the body is kept at uniform temperature, $T_{w}$, situated in ambient dusty fluid of undisturbed temperature, $T_{\infty}$, such that $T_{w}>T_{\infty}$.

- The dust particles are assumed to have same size and shape and uniformly distributed in the carrier fluid. Furthermore, the size of the solid particles is large enough to neglect the Brownian motion.

- The concentration of dust particles is uniform in time and space and "dilute" in the sense that the colliding forces between the solid particles are insignificant. Therefore, the solid spherical particles of equal radii are assumed to be non-interacting in the analysis.

Based on the above-mentioned assumptions, the governing boundary layer equations for two-phase dusty viscous incompressible fluid can be written in the underlying form (see Ref. $[1-3,14])$.

For the gas phase:

$$
\begin{gathered}
\frac{\partial u}{\partial x}+\frac{\partial v}{\partial y}+\frac{u}{r} \frac{d r}{d x}=0 \\
\rho\left(u \frac{\partial u}{\partial x}+v \frac{\partial u}{\partial y}-\frac{w^{2}}{r} \frac{d r}{d x}\right)=\mu \frac{\partial^{2} u}{\partial y^{2}}+\rho g \beta\left(T-T_{\infty}\right)+\frac{\rho_{p}}{\tau_{m}}\left(u_{p}-u\right) \\
\rho\left(u \frac{\partial w}{\partial x}+v \frac{\partial w}{\partial y}+\frac{u w}{r} \frac{d r}{d x}\right)=\mu \frac{\partial^{2} w}{\partial y^{2}}+\frac{\rho_{p}}{\tau_{m}}\left(w_{p}-w\right) \\
\rho c_{p}\left(u \frac{\partial T}{\partial x}+v \frac{\partial T}{\partial y}\right)=\kappa \frac{\partial^{2} T}{\partial y^{2}}+\frac{\rho_{p} c_{s}}{\tau_{T}}\left(T_{p}-T\right)
\end{gathered}
$$

For the particle phase:

$$
\begin{gathered}
\frac{\partial u_{p}}{\partial x}+\frac{\partial v_{p}}{\partial y}+\frac{u_{p}}{r} \frac{d r}{d x}=0 \\
\rho_{p}\left(u_{p} \frac{\partial u_{p}}{\partial x}+v_{p} \frac{\partial u_{p}}{\partial y}-\frac{w_{p}^{2}}{r} \frac{d r}{d x}\right)=-\frac{\rho_{p}}{\tau_{m}}\left(u_{p}-u\right) \\
\rho_{p}\left(u_{p} \frac{\partial w_{p}}{\partial x}+v_{p} \frac{\partial w_{p}}{\partial y}+\frac{u_{p} w_{p}}{r} \frac{d r}{d x}\right)=-\frac{\rho_{p}}{\tau_{m}}\left(w_{p}-w\right) \\
\rho_{p} c_{p}\left(u_{p} \frac{\partial T_{p}}{\partial x}+v_{p} \frac{\partial T_{p}}{\partial y}\right)=-\frac{\rho_{p} c_{s}}{\tau_{T}}\left(T_{p}-T\right)
\end{gathered}
$$

where $T, p, \rho, c_{p}, \beta, \kappa$ and $\mu$ are respectively the temperature, pressure, density, specific heat at constant pressure, volumetric expansion coefficient, thermal conductivity and coefficient of viscosity of the fluid/carrier phase. $g$ is the $x$-component of the local gravitational acceleration vector in the direction of increasing $x$. In the analysis, $(x, y, z)$ are the components of the curvilinear coordinate system, $u$ the component of the velocity in the surface-contour 
direction, $v$ the velocity component in the normal direction, $w$ the tangential component of the velocity in rotating $z$ direction. Similarly, $\left(u_{p}, v_{p}, w_{p}\right), T_{p}, p_{p}, \rho_{p}$ and $c_{s}$ corresponds to the velocity vector, temperature, pressure, density and specific heat for the particle phase. In addition, $r(x)$ is the radial distance from axis of symmetry to the axial direction and $\tau_{m}$ $\left(\tau_{T}\right)$ the momentum relaxation time (thermal relaxation time) during which the velocity (temperature) of the particle phase relative to the fluid is reduced to $1 / e$ times its initial value. In order to determine the flow fields of the fluid and the dust particles, the boundary layer equations stated above are to be solved under appropriate boundary conditions. These are:

$$
\begin{gathered}
u=v=0, \quad w=r \Omega, \quad T=T_{w} \text { at } y=0 \\
u \rightarrow 0, \quad w \rightarrow 0, \quad T \rightarrow T_{\infty} \text { as } y \rightarrow \infty \\
u_{p}=v_{p}=0, \quad w_{p}=r \Omega, \quad T_{p}=T_{w} \text { at } y=0 \\
u_{p} \rightarrow 0, \quad w_{p} \rightarrow 0, \quad T_{p} \rightarrow T_{\infty} \text { as } y \rightarrow \infty
\end{gathered}
$$

The system of conservation equations can be recast into a dimensionless form by using the appropriate stretched coordinates, a reference length $L$ and a reference velocity $U_{\infty}=L \Omega$. Therefore, introducing the following scaling and dimensionless numbers:

$$
\begin{aligned}
& \bar{x}=\frac{x}{L}, \quad \bar{y}=\frac{R e^{1 / 2} y}{L}, \quad\left(\bar{u}, \bar{u}_{p}\right)=\frac{\left(u, u_{p}\right)}{U_{\infty}}, \quad\left(\bar{v}, \bar{v}_{p}\right)=\frac{R e^{1 / 2}}{U_{\infty}}\left(v, v_{p}\right), \quad\left(\bar{w}, \bar{w}_{p}\right)=\frac{\left(w, w_{p}\right)}{U_{\infty}} \\
& g=g_{x} K(\bar{x}), \quad \bar{r}=\frac{r}{L}, \quad\left(\theta, \theta_{p}\right)=\frac{\left(T, T_{p}\right)-T_{\infty}}{T_{w}-T_{\infty}}, \quad \operatorname{Re}=\frac{U_{\infty} L}{\nu}, \quad G r=\frac{g_{x} \beta\left(T_{w}-T_{\infty}\right) L^{3}}{\nu^{2}}, \\
& \tau_{T}=\frac{3 \gamma \tau_{m} \operatorname{Pr}}{2}, \quad \lambda=\frac{G r}{R e^{2}}, \quad \operatorname{Pr}=\frac{\nu}{\alpha}, \quad D_{\rho}=\frac{\rho_{p}}{\rho}, \quad \gamma=\frac{c_{s}}{c_{p}}, \quad \alpha_{d}=\frac{L}{U_{\infty} \tau_{m}}
\end{aligned}
$$

into the Eqs. (1)-(10), the normalized continuity, momentum and temperature equations will be transformed in underlying form.

For gas phase:

$$
\begin{gathered}
\frac{\partial \bar{u}}{\partial \bar{x}}+\frac{\partial \bar{v}}{\partial \bar{y}}+\frac{\bar{u}}{\bar{r}} \frac{d \bar{r}}{d \bar{x}}=0 \\
\left(\bar{u} \frac{\partial \bar{u}}{\partial \bar{x}}+\bar{v} \frac{\partial \bar{u}}{\partial \bar{y}}-\frac{\bar{w}^{2}}{\bar{r}} \frac{d \bar{r}}{d \bar{x}}\right)=\frac{\partial^{2} \bar{u}}{\partial \bar{y}^{2}}+\lambda K(\bar{x}) \theta+D_{\rho} \alpha_{d}\left(\bar{u}_{p}-\bar{u}\right) \\
\left(\bar{u} \frac{\partial \bar{w}}{\partial \bar{x}}+\bar{v} \frac{\partial \bar{w}}{\partial \bar{y}}+\frac{\bar{u} \bar{w}}{\bar{r}} \frac{d \bar{r}}{d \bar{x}}\right)=\frac{\partial^{2} \bar{w}}{\partial \bar{y}^{2}}+D_{\rho} \alpha_{d}\left(\bar{w}_{p}-\bar{w}\right) \\
\left(\bar{u} \frac{\partial \theta}{\partial \bar{x}}+\bar{v} \frac{\partial \theta}{\partial \bar{y}}\right)=\frac{1}{\operatorname{Pr}}\left(\frac{\partial^{2} \theta}{\partial \bar{y}^{2}}+\frac{2}{3} D_{\rho} \alpha_{d}\left(\theta_{p}-\theta\right)\right)
\end{gathered}
$$

For particle phase:

$$
\begin{gathered}
\frac{\partial \bar{u}_{p}}{\partial \bar{x}}+\frac{\partial \bar{v}_{p}}{\partial \bar{y}}+\frac{\bar{u}_{p}}{\bar{r}} \frac{d \bar{r}}{d \bar{x}}=0 \\
\left(\bar{u}_{p} \frac{\partial \bar{u}_{p}}{\partial \bar{x}}+\bar{v}_{p} \frac{\partial \bar{u}_{p}}{\partial \bar{y}}-\frac{\bar{w}_{p}^{2}}{\bar{r}} \frac{d \bar{r}}{d \bar{x}}\right)=-\alpha_{d}\left(\bar{u}_{p}-\bar{u}\right) \\
\left(\bar{u}_{p} \frac{\partial \bar{w}_{p}}{\partial \bar{x}}+\bar{v}_{p} \frac{\partial \bar{w}_{p}}{\partial \bar{y}}+\frac{\bar{u}_{p} \bar{w}_{p}}{\bar{r}} \frac{d \bar{r}}{d \bar{x}}\right)=-\alpha_{d}\left(\bar{w}_{p}-\bar{w}\right)
\end{gathered}
$$




$$
\left(\bar{u}_{p} \frac{\partial \theta_{p}}{\partial \bar{x}}+\bar{v}_{p} \frac{\partial \theta_{p}}{\partial \bar{y}}\right)=-\frac{2}{3 \gamma \operatorname{Pr}} \alpha_{d}\left(\theta_{p}-\theta\right)
$$

In the above system, $\lambda, G r$, Pr are respectively the buoyancy parameter, Grashof number and Prandtl number. The gravitational acceleration for the round nosed geometry is defined as $g=g_{x} K(\bar{x})$, where $K(\bar{x})$ is non-dimensional function of $\bar{x}$. The carrier phase and particle phase are interacted through the specific heat ratio parameter, $\gamma$, mass concentration parameter, $D_{\rho}$, and dust parameter, $\alpha_{d}$. The detailed description of these interactions parameter is given in Refs. [5] and [14].

The boundary conditions for the gas phase:

$$
\begin{gathered}
\bar{u}=\bar{v}=0, \quad \bar{w}=\bar{r}, \quad \theta=1 \quad \text { at } \bar{y}=0, \\
\bar{u} \rightarrow 0, \quad \bar{w} \rightarrow 0, \quad \theta \rightarrow 0 \quad \text { as } \quad \bar{y} \rightarrow \infty
\end{gathered}
$$

For the particle phase:

$$
\begin{gathered}
\bar{u}_{p}=\bar{v}_{p}=0, \quad \bar{w}_{p}=\bar{r}, \theta_{p}=1 \quad \text { at } \bar{y}=0, \\
\bar{u}_{p} \rightarrow 0, \quad \bar{w}_{p} \rightarrow 0, \quad \theta_{p} \rightarrow 0 \quad \text { as } \bar{y} \rightarrow \infty,
\end{gathered}
$$

The above dimensionless system of equations is further transformed into another coordinate system by means of some continuous transformations as defined below:

$$
\begin{aligned}
& \left(\bar{u}, \bar{u}_{p}\right)=\bar{r}\left(U, U_{p}\right), \quad\left(\bar{w}, \bar{w}_{p}\right)=\bar{r}\left(W, W_{p}\right), \quad\left(\bar{v}, \bar{v}_{p}\right)=\bar{r}^{2}(2 X)^{-1 / 2}\left(V, V_{p}\right) \\
& \theta(\bar{x}, \bar{y})=\Theta(X, Y), \quad X=\int_{0}^{\bar{x}}[\bar{r}(\bar{x})]^{3} d \bar{x}, \quad Y=\bar{r}^{2}(2 X)^{-1 / 2} \bar{y}
\end{aligned}
$$

The system of Eqs.(12)-(21) becomes as follows:

$$
\begin{gathered}
2 P(\bar{x}) U+2 X \frac{\partial U}{\partial X}+(2 P(\bar{x})-1) Y \frac{\partial U}{\partial Y}+\frac{\partial V}{\partial Y}=0 \\
2 X U \frac{\partial U}{\partial X}+(V+(2 P(\bar{x})-1) Y U) \frac{\partial U}{\partial Y}+P(\bar{x})\left(U^{2}-W^{2}\right)=\frac{\partial^{2} U}{\partial Y^{2}}+\lambda Q(\bar{x}) \Theta \\
+R(\bar{x}) D_{\rho} \alpha_{d}\left(U_{p}-U\right) \\
2 X U \frac{\partial W}{\partial X}+(V+(2 P(\bar{x})-1) Y U) \frac{\partial W}{\partial Y}+2 P(\bar{x}) U W=\frac{\partial^{2} W}{\partial Y^{2}}+R(\bar{x}) D_{\rho} \alpha_{d}\left(W_{p}-W\right) \\
2 X U \frac{\partial \Theta}{\partial X}+(V+(2 P(\bar{x})-1) Y U) \frac{\partial \Theta}{\partial Y}=\frac{1}{\operatorname{Pr}}\left(\frac{\partial^{2} \Theta}{\partial Y^{2}}+\frac{2}{3} D_{\rho} \alpha_{d}\left(\Theta_{p}-\Theta\right)\right) \\
2 P(\bar{x}) U_{p}+2 X \frac{\partial U_{p}}{\partial X}+(2 P(\bar{x})-1) Y \frac{\partial U_{p}}{\partial Y}+\frac{\partial V_{p}}{\partial Y}=0 \\
2 X U_{p} \frac{\partial U_{p}}{\partial X}+\left(V_{p}+(2 P(\bar{x})-1) Y U_{p}\right) \frac{\partial U_{p}}{\partial Y}+P(\bar{x})\left(U_{p}^{2}-W_{p}^{2}\right)=-R(\bar{x}) \alpha_{d}\left(U_{p}-U\right) \\
2 X U_{p} \frac{\partial W_{p}}{\partial X}+\left(V_{p}+(2 P(\bar{x})-1) Y U_{p}\right) \frac{\partial W_{p}}{\partial Y}+2 P(\bar{x}) U_{p} W_{p}=-R(\bar{x}) \alpha_{d}\left(W_{p}-W\right) \\
2 X U_{p} \frac{\partial \Theta_{p}}{\partial X}+\left(V_{p}+(2 P(\bar{x})-1) Y U_{p}\right) \frac{\partial \Theta_{p}}{\partial Y}=-\frac{2}{3 \gamma \operatorname{Pr}} \alpha_{d}\left(\Theta_{p}-\Theta\right)
\end{gathered}
$$


The transformed boundary conditions are:

$$
\begin{gathered}
U=V=U_{p}=V_{p}=0, \quad W=\Theta=W_{p}=\Theta_{p}=1, \quad \text { at } Y=0, \\
U=U_{p}=0, \quad W=\Theta=W_{p}=\Theta_{p}=0, \quad \text { as } Y \rightarrow \infty
\end{gathered}
$$

Round nosed bodies are of great interest due to the reason that i) they have been proposed as necessary for adequate radar installation and ii) to derive optimum shapes mathematically that have a small blunt region at the tip. For the problem under consideration, we discuss the application of the results for a rotating hemisphere. The radius of the rotating hemisphere is assumed to be $R$, which is oriented parallel with respect to the gravitational vector $\mathbf{g}$. If we select $R$ as a reference characteristic length, i.e. $R=L$, then we have the following dimensionless variables:

$$
\bar{r}(\bar{x})=K(\bar{x})=\sin (\bar{x}), \quad X=(1 / 3)\left(\cos ^{3} \bar{x}-3 \cos \bar{x}+2\right)
$$

Therefore for the case of hemisphere, $P(\bar{x})$ and $Q(\bar{x})$ are found to be:

$$
P(\bar{x})=\frac{2 \cos \bar{x}\left(\cos ^{3} \bar{x}-3 \cos \bar{x}+2\right)}{3 \sin ^{4} \bar{x}}, Q(\bar{x})=\frac{2\left(\cos ^{3} \bar{x}-3 \cos \bar{x}+2\right)}{3 \sin ^{4} \bar{x}}
$$

The Local skin friction coefficient and local rate of heat transfer are of prime interest in many technological and engineering applications. These dimensionless quantities can be expressed mathematically as:

$$
\tau_{x}=(2 X)^{-1 / 2} \bar{r}^{3}\left(\frac{\partial U}{\partial Y}\right)_{Y=0}, \quad Q=-(2 X)^{-1 / 2} \bar{r}^{2}\left(\frac{\partial \Theta}{\partial Y}\right)_{Y=0}
$$

The detailed numerical solutions for the coupled system of non-linear partial differential Eqs. (23)-(30) subject to the boundary conditions (31) are obtained through implicit finite difference method, since the computational grids can be fitted to the body shape in $(X, Y)$ coordinates. The system of equations is discretized by exploiting the central difference quotients for diffusion terms and the forward difference for the convection terms. The computational process is started at $X=0.0$ and at every $X$ station, the computations are iterated until the difference of the results, of two successive iterations become less or equal to $10^{-6}$. In order to get accurate results, we have compared the results at different grid size in $Y$ direction and reached at the conclusion to chose $\Delta Y=0.064$. The maximum value of $Y$ is taken to be 90.0. A detail description of discretization procedure and numerical scheme is presented in [19].

\section{Results and Discussion}

Numerical results are presented in terms of skin friction coefficient, local rate of heat transfer, velocity profiles and temperature profiles for the case of a rotating hemisphere. For this, numerical computations are made for some practical examples, such that, i) metal particles in gas $\left(D_{\rho}=10^{2}, \operatorname{Pr}=0.7, \gamma=0.45\right)$ and ii) metal particles in water $\left(D_{\rho}=10, \operatorname{Pr}=7.0\right.$, $\gamma=0.1)$ by taking $\alpha_{d}=(1.0,2.0,3.0)$ and $\lambda=(0.0,0.1,0.5,1.0)$ (for details see [20]). The numerical values of $\tau_{x}$ and $Q$ for air-particulate suspension are entered in Table 1 by taking the value of buoyancy ratio parameter as $\lambda=(0.0,1.0)$. As it can be clearly seen from the numerical values of $\tau_{x}$ and $Q$ at $Y=0$, that both of the physical quantities increase 
by loading the dust particles in pure fluid (i.e, $D_{\rho}=0.0$ ). The rate of heat transfer gets stronger in the vicinity of the heated hemisphere for $D_{\rho}=100.0$. The quantitative data also show the influence of buoyancy ratio parameter $\lambda$ on $\tau_{x}$ and $Q$. The values of $\tau_{x}$ and $Q$ increase in magnitude when $\lambda$ increases from 0.0 to 1.0, that reveals the fact that the local skin friction and rate of heat transfer are more pronounced for free convective regime.

Table 1: Numerical values of $\tau_{x}$ and $Q$ for $D_{\rho}=0.0,100.0, \gamma=0.45, \lambda=0.0,1.0, \operatorname{Pr}=0.7$ and $\alpha_{d}=1.0$

\begin{tabular}{|c|c|c|c|c|}
\hline \multirow{2}{*}{$X$} & \multicolumn{2}{|c|}{$\tau_{x}$} & \multicolumn{2}{c|}{$Q$} \\
\cline { 2 - 5 } & \multicolumn{4}{|c|}{$\lambda=0.0$} \\
\cline { 2 - 5 } & $D_{\rho}=0.0$ & $D_{\rho}=100.0$ & $D_{\rho}=0.0$ & $D_{\rho}=100.0$ \\
\hline 0.1 & 0.05082 & 0.17881 & 0.32316 & 1.96283 \\
0.2 & 0.10041 & 0.35652 & 0.32126 & 1.95203 \\
0.3 & 0.14736 & 0.53126 & 0.31809 & 1.93399 \\
0.4 & 0.19042 & 0.70146 & 0.31364 & 1.90861 \\
0.5 & 0.22840 & 0.86546 & 0.30790 & 1.87579 \\
\hline & \multicolumn{4}{|c|}{$\lambda=1.0$} \\
\hline 0.1 & 0.10749 & 0.18937 & 0.48800 & 2.06113 \\
0.2 & 0.21376 & 0.37759 & 0.48649 & 2.05096 \\
0.3 & 0.31719 & 0.56274 & 0.48398 & 2.03399 \\
0.4 & 0.41642 & 0.74320 & 0.48046 & 2.01012 \\
0.5 & 0.51018 & 0.91723 & 0.47594 & 1.97924 \\
\hline
\end{tabular}

In order to validate the accuracy of our scheme and numerical computations, comparison is also being made with already available data in literature. It is rather important to mention here that for $\lambda=R_{d}=D_{\rho}=0.0$, the analysis of Suwono [1] and Hossain et al. [3] become special cases of the present analysis. In former study, the authors obtained the numerical solutions of the governing equations with the help of Runge-Kutta-Merson (RKM) method near the leading edge where axial coordinate is considered small, while in the later analysis, the authors used Keller box method to obtain solutions over the whole range of axial coordinate. The present computational results, however, are obtained for the same range of axial coordinate $X$, from implicit finite difference method. In-spite of different formulations and methods as well; the computational results are compared in Fig. 2 by keeping the physical parameters as: $\lambda=0.1,0.5,1.0, R_{d}=0.0, \operatorname{Pr}=0.72$ and $D_{\rho}=0.0$ for the interval $[0, \pi / 2]$. The comparison shows that the results obtained by implicit finite difference method are in a good agreement with those obtained by RKM and Keller box method. Therefore, it can be concluded from Fig. 2 that present numerical scheme give accurate results.

Graphical presentation of $\tau_{x}$ and $Q$ is given in Fig. 3 for air and water particle mixtures. It is worth mentioning that the curves in which air is acting as a carrier fluid exhibit considerable change due to the presence of metal particles as compared to water particulate suspension. In Fig. 3(a), it can be seen that both the skin friction and heat transfer rate increase by increasing the value of mass concentration parameter $D_{\rho}$. Such behavior is expected because the carrier fluid gains the kinetic and thermal energy by 
interacting with the dust particles and this leads to an increase of the velocity of carrier fluid as compared to pure fluid case. Ultimately, the velocity gradient for the carrier fluid increases at the surface of the rotating hemisphere. Particularly, it can be seen from Fig. 3(b) that the rate of heat transfer drastically increases by increasing the mass concentration parameter, $D_{\rho}$, for air particulate suspension. In this case, the dusty air gains some thermal energy from particles and the temperature of the carrier fluid increases, which ultimately promotes the rate of heat transfer.

Variation of dust parameter is analyzed in Fig. 4 by setting: $\alpha_{d}=1.0,2.0,3.0$ for air particulate suspension. It is interesting to infer from Fig. 4(a), that the value of skin friction coefficient, $\tau_{x}$, increases owing to the increase in dust parameter, $\alpha_{d}$. The presence of inert particles is responsible for the enhancement of skin friction at the surface. The rate of heat transfer remains almost invariant by intensifying the values of $\alpha_{d}$. It has also been observed from numerical computations, that the rate of heat transfer remains uniform if $\alpha_{d} \geq 3.0$.

Contribution of buoyancy parameter $\lambda$ on $\tau_{x}$ and $Q$ is shown in Fig. 5. The problem is termed as i) assisting buoyancy flow for $\lambda>0$, ii) opposing buoyancy flow for $\lambda<0$ and iii) purely forced convection flow if $\lambda=0$. In the present situation, it is observed that both physical quantities, namely, friction factor and heat transfer rate get promoted at the surface of the rotating hemisphere when buoyancy parameter is increased. Therefore, positive value of $\lambda$ will assist the flow as expected. As a result, boundary layer becomes thinner and the skin friction coefficient and the heat transfer coefficient appear more pronounced within the boundary layer region.

The effect of mass concentration parameter on velocity and temperature profiles for both phases is illustrated in Fig. 6. For comparison, suspension without particle cloud (pure air) is also presented. The curve in Fig. 6 shows that, by loading the dust particles in the air mixture, the velocity and temperature of both phases diminish considerably. For the non-zero value of $D_{\rho}$, the velocity and temperature profiles of carrier and dusty phase decays quickly to asymptotical values and approach to zero in the free stream region. The presence of dust particles is responsible for this behavior, as they extensively resist the flow and promote the frictional forces, which results in reduction of velocity and temperature of the dusty phase. Therefore, $U_{p}, \Theta_{p}, W_{p}$ rapidly attain their limiting values as compared to $U, \Theta, W$.

Representative velocity and temperature profiles for carrier as well as dusty phase under the effect of parameter $\lambda$ are displayed in Fig. 7. It can be seen from Fig. 7(a), that the non-zero values of $\lambda$ acts like a supportive driving force that accelerates the air particulate suspension flow and as a result the velocity for both phases $U, U_{p}$, within the boundary layer increases significantly. For small values of $\lambda$, the flow approaches the forced convective regime and gradually, as $\lambda$ increases, the flow becomes fully developed and the convection mode changes from the forced to the natural convection flow. Furthermore, it can be seen from Figs. 7(b) and 7(c) the temperature profile, $\Theta$, and velocity component $W$ profile decreases with an increase in the value of $\lambda$. It is therefore concluded that greater the values of $\lambda$ more quickly an asymptotic behavior is attained. 


\section{Conclusions}

The present analysis aims to compute the numerical results of two-phase boundary layer flow of dusty fluid induced by an axi-symmetric round nosed body. As an example, the solutions are presented for the case of rotating hemisphere. Coordinate transformations (Primitive variable formulations) are applied to switch the governing equations of the carrier and the dispersed phase into another set of equations. Two-point finite difference solutions are obtained for the semi-infinite domain. Effect of various emerging parameters are explored by expressing their relevance on skin friction coefficient and the rate of heat transfer. Velocity and temperature distributions are plotted for carrier as well as particle phase. From this analysis, it is observed that mass concentration parameter, $D_{\rho}$, and the buoyancy ratio parameter, $\lambda$, promote the skin friction coefficient as well as the rate of heat transfer, whereas, the dust parameter, $\alpha_{d}$, has a pronounced effect in the reduction of skin friction within the boundary layer region. In addition, the velocity and temperature profiles for both phases of air quickly attain an asymptotic behavior when $D_{\rho}$ is penetrated into the mechanism.

\section{References}

[1] Suwono, A., Buoyancy effects on flow and heat transfer on rotating axi-symmetric round-nosed bodies, Int. J. Heat Mass Transfer, 23, 1980, 819-831.

[2] Hossain, M. A., Das, S. K., Pop, I., MHD free convection flow near rotating axisymmetric round-nosed bodies, Magnitnaya Gidrodinamika, 2, 1996, 68-73.

[3] Hossain, M. A., Anghel, A., Pop, I., Thermal radiation effects on free convection over a rotating axi-symmetric body with application to rotating hemispehere, Arch. Mech., 54, 2002, 55-74.

[4] Siddiqa, S., Begum, N., Hossain, M. A., Gorla, R. S. R., Influence of temperaturedependent density on flow near rotating axi-symmetric round-nosed body, submitted.

[5] Rudinger, G., Fundamentals of gas-particle flow, Elsevier Scientific Publishing Co., Amsterdam, 1980.

[6] Farbar, L., Morley, M. J., Heat transfer to flowing gas-solid mixtures in a circular tube, Ind. Eng. Chem., 49, 1957, 1143-1150.

[7] Marble, F. E., Dynamics of a gas containing small solid particles, combustion and propulsion, 5th AGARD colloquium, Pergamon press, 1963.

[8] Singleton, R. E., Fluid mechanics of gas-solid particle flow in boundary layers, Ph.D. Thesis, California Institute of Technology, 1964.

[9] Michael, D. H., Miller, D. A., Plane parallel flow of a dusty gas, Mathematica, 13, 1966, 97-109.

[10] Saffman, P. G., On the stability of laminar flow of a dusty gas, J. Fluid Mech., 13, 1962, 120-128. 
[11] Michael, D. H., The steady motion of a sphere in a dusty gas,J. Fluid Mech., 31, 1968, 175-192.

[12] Datta, N., Mishra, S. K., Boundary layer flow of a dusty fluid over a semi-infinite flat plate, Acta Mech., 42, 1982, 71-83.

[13] Agranat, V. M., Effect of pressure gradient of friction and heat transfer in a dusty boundary layer, Fluid Dyn., 23, 1988, 729-732.

[14] Siddiqa, S., Hossain, M. A., Saha, S. C., Two-phase natural convection flow of a dusty fluid, Int. J. Numer. Method, 25, 2015, 1542- 1556.

[15] Siddiqa, S., Abrar, M. N., Hossain, M. A., Awais, M., Dynamics of two-phase dusty fluid flow along a wavy surface, Int. J. Nonlinear Sci. Numer. Simul., 17, 2016, 185193.

[16] Siddiqa, S., Begum, N., Hossain, M. A., Compressible dusty gas along a vertical wavy surface, Appl. Math. Comput., 293, 2017, 600-610.

[17] Siddiqa, S., Begum, N., Hossain, M. A., Mustafa, N., Gorla, R. S. R., Two phase dusty fluid flow along a cone with variable properties, Heat Mass Transf., DOI 10.1007/s00231-016-1918-y.

[18] Siddiqa, S., Begum, N., Hossain, M. A., Massarotti, N., Influence of thermal radiation on contaminated air and water flow past a vertical wavy frustum of a cone, Int. Commun. Heat Mass Transf., 76, 2016, 63-68.

[19] Siddiqa, S., Begum, N., Hossain, M. A., Radiation effects from an isothermal vertical wavy cone with variable fluid properties, Appl. Math. Comput., 289, 2016, 149-158.

[20] Apazidis, N., Temperature distribution and heat transfer in a particle-fluid flow past a heated horizontal plate, Int. J. Multiphase Flow, 16, 1990, 495-513. 


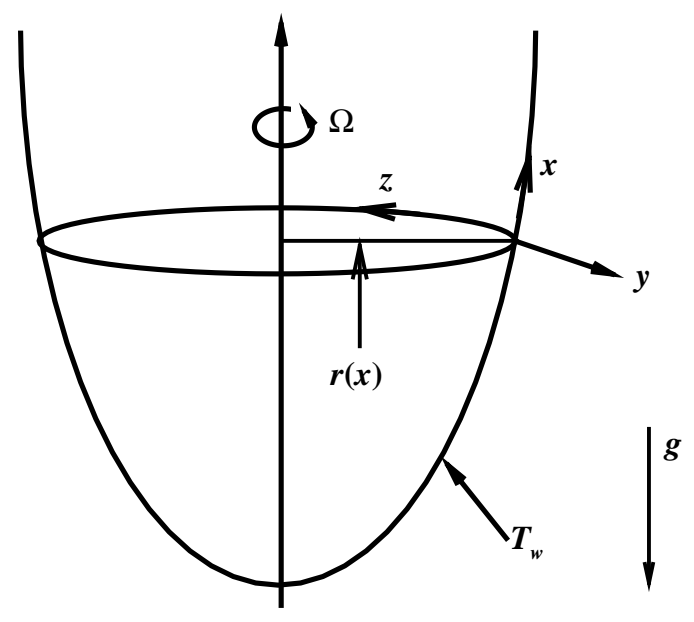

Fig. 1 Physical Model
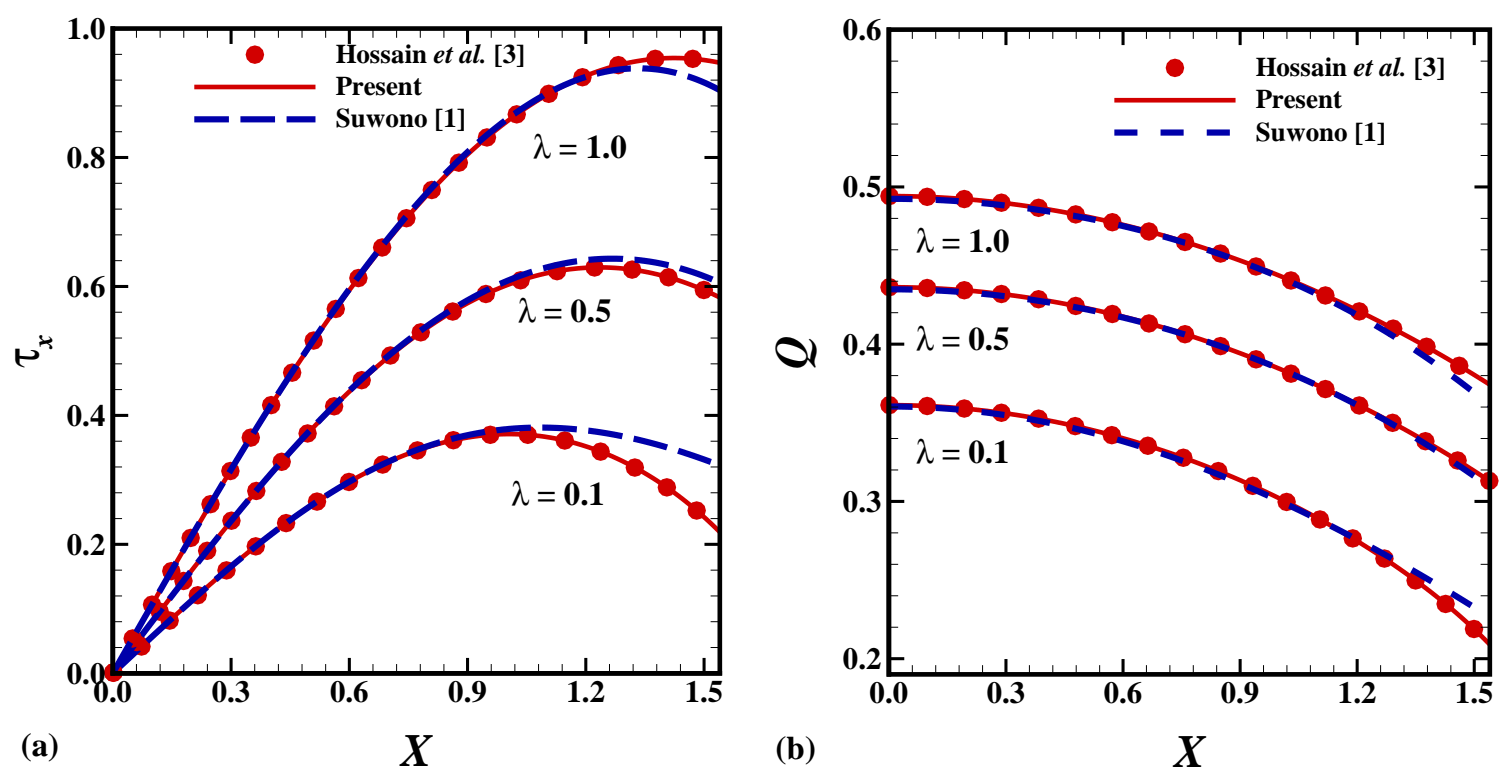

Fig. 2 Comparison of (a) $\tau_{x}$ and (b) $Q$ without dust particles $\left(D_{\rho}=0.0\right)$ obtained for $\lambda=0.1,0.5,1.0$ and $\operatorname{Pr}=0.72$. 

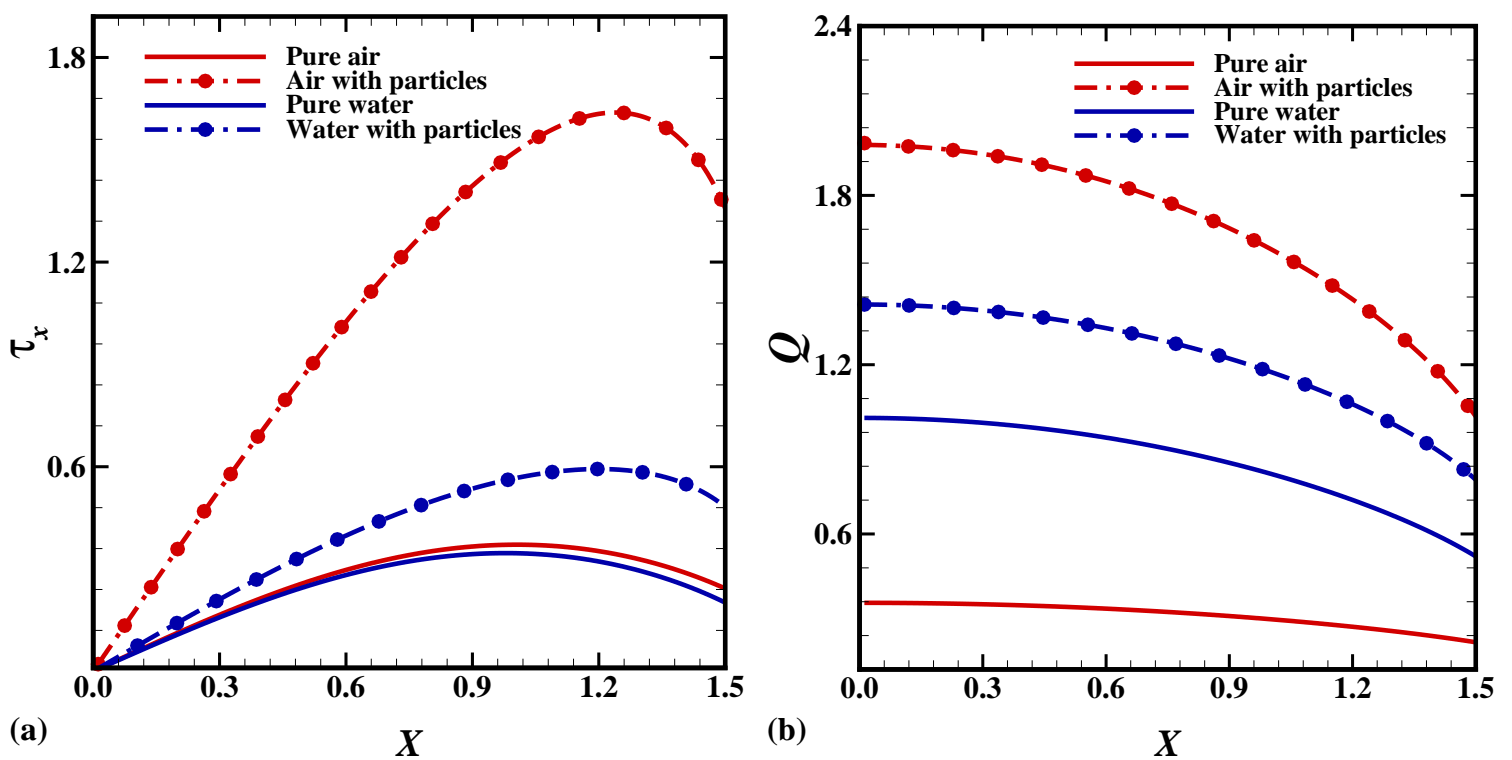

Fig. 3 (a) $\tau_{x}$ and (b) $Q$ for air and water particle flow with $D_{\rho}=100.0$ (air),

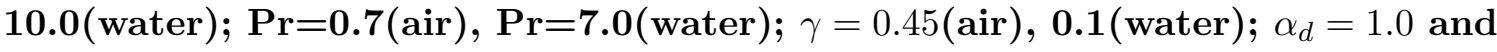
$\lambda=0.1$.
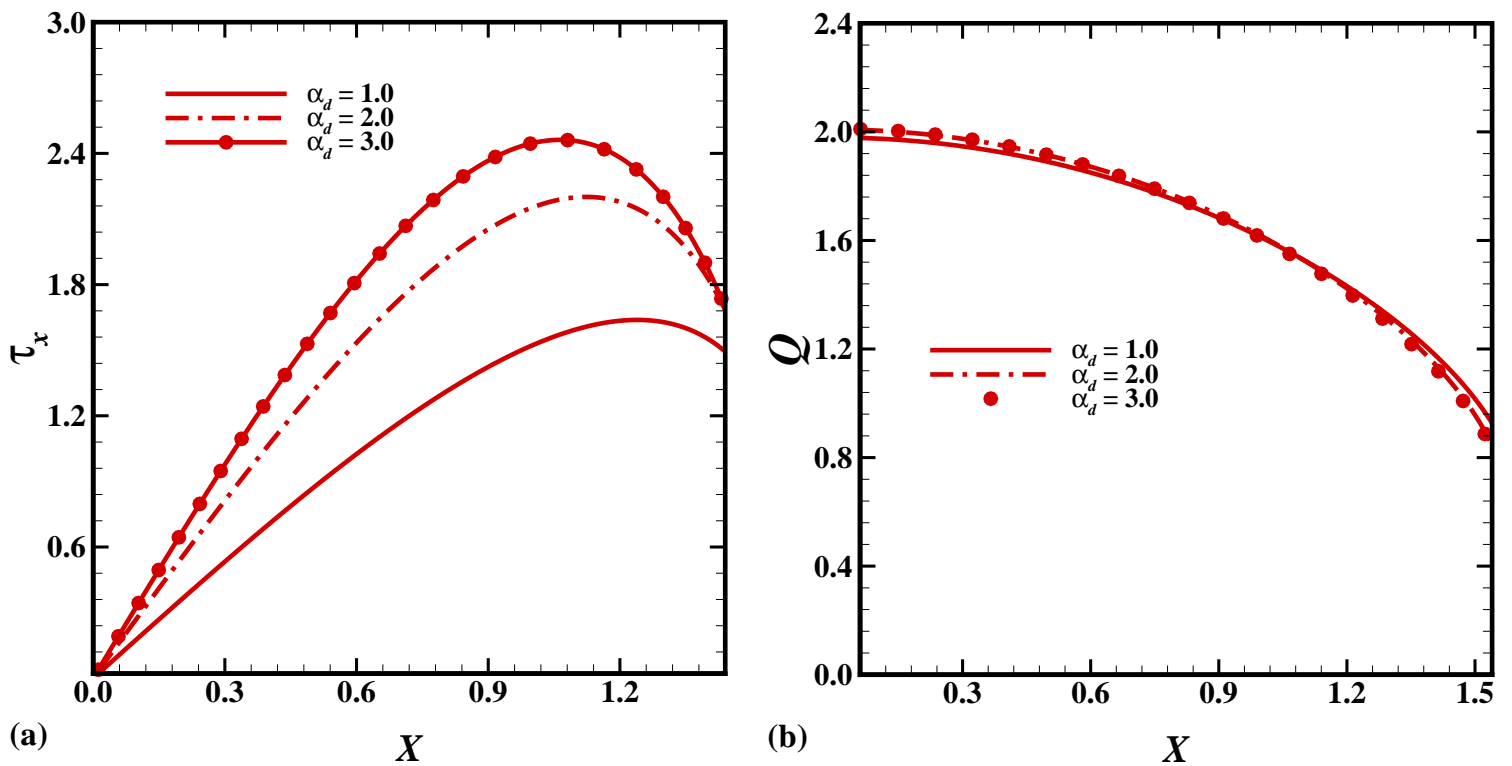

Fig. 4 (a) $\tau_{x}$ and (b) $Q$ for $\alpha_{d}=1.0,2.0,3.0$ while $D_{\rho}=100.0, \operatorname{Pr}=0.7, \gamma=0.45$ and $\lambda=0.1$ 

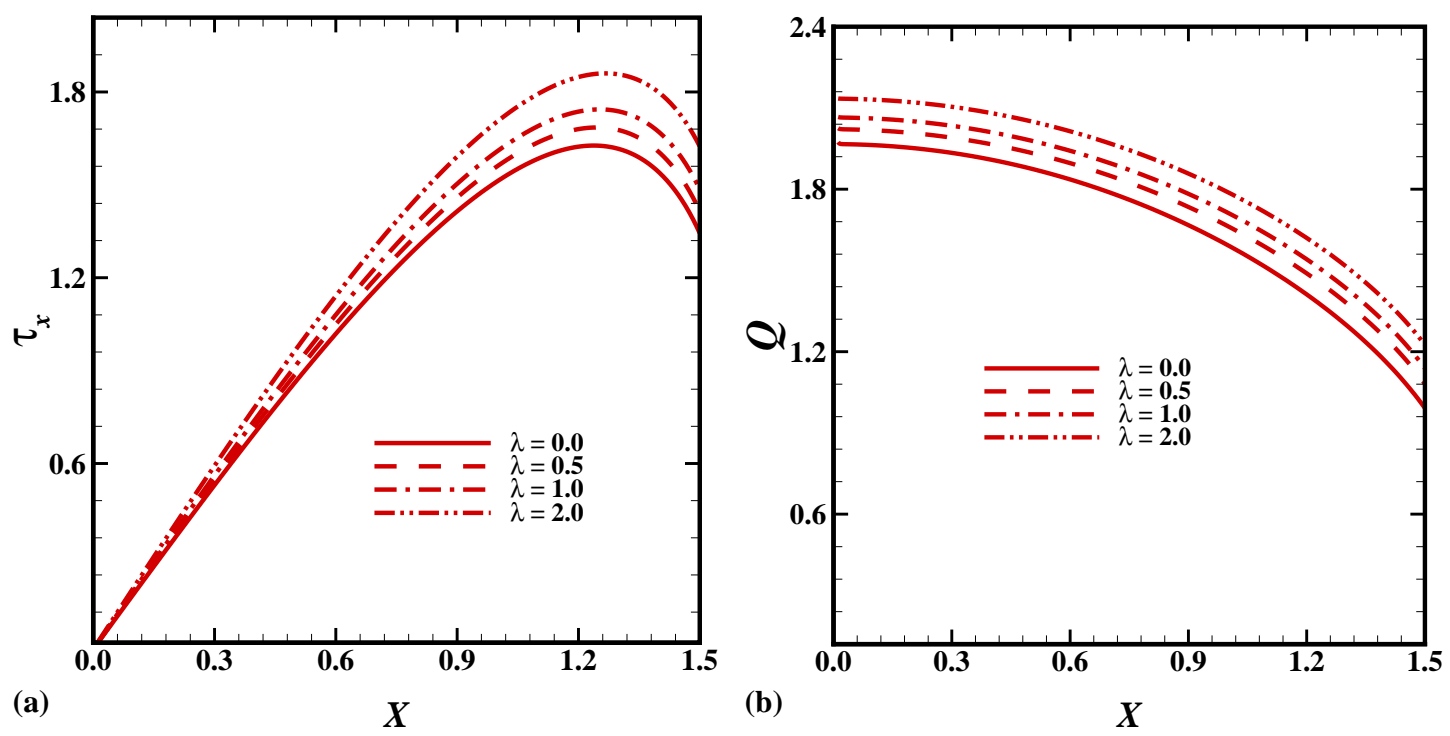

Fig. 5 (a) $\tau_{x}$ and (b) $Q$ for $\lambda=0.0,0.5,1.0,2.0$ while $D_{\rho}=100.0, \operatorname{Pr}=0.7, \gamma=0.45$ and $\alpha_{d}=1.0$. 

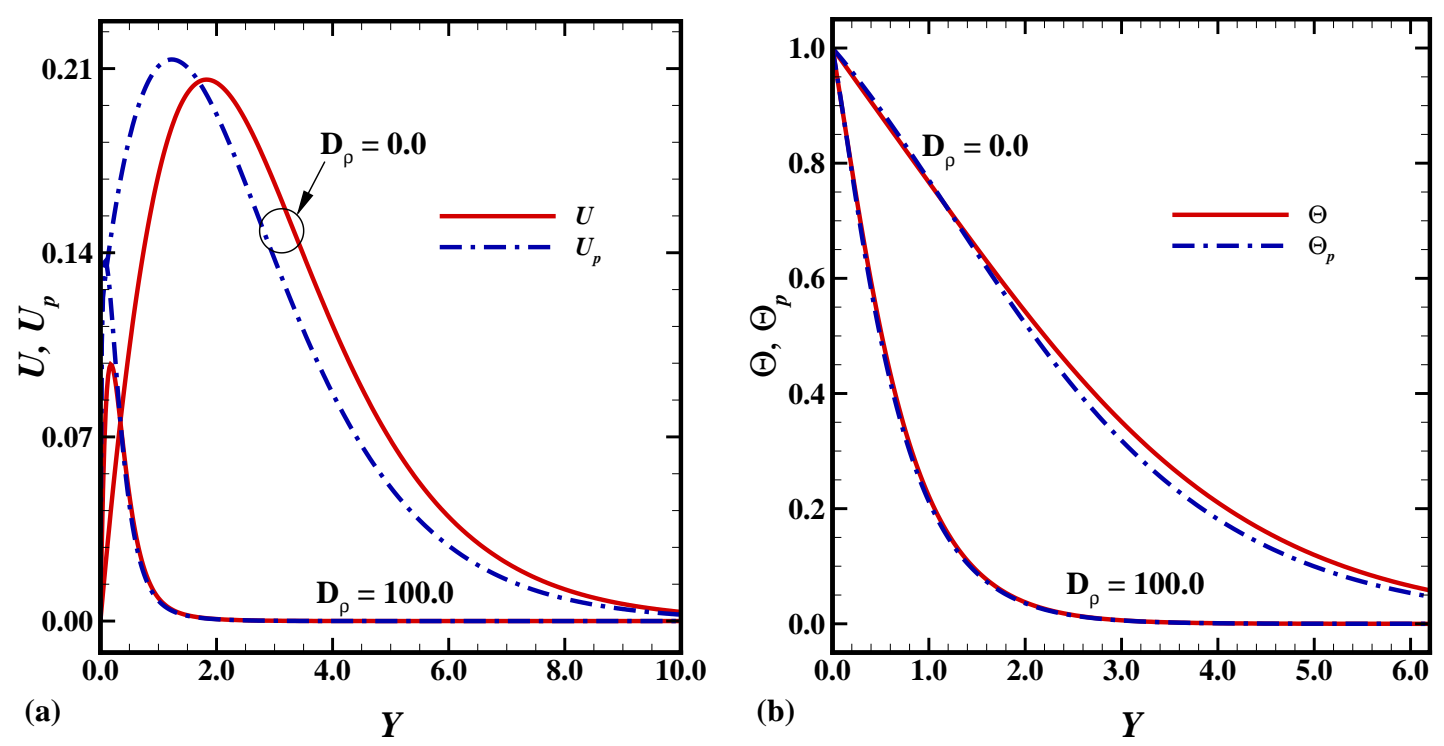

(a)

$$
\boldsymbol{Y}
$$

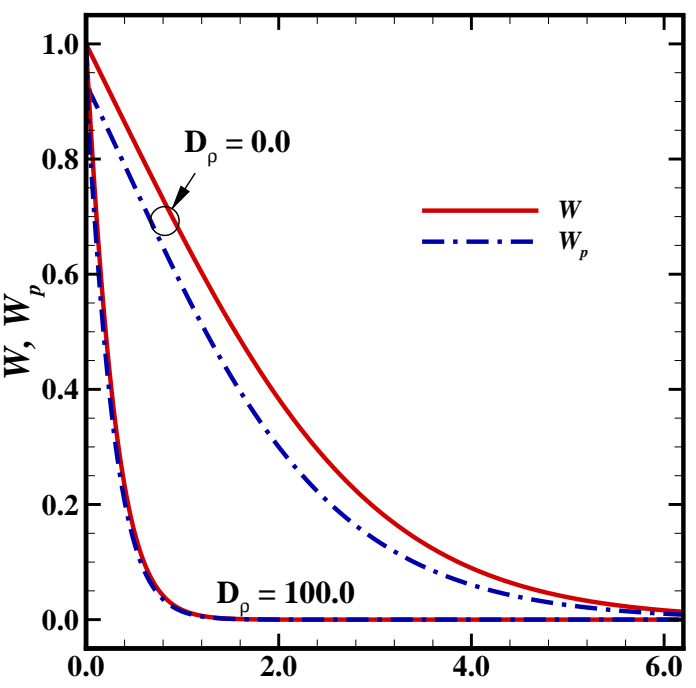

(c)

$\boldsymbol{Y}$

Fig. 6 (a) Velocity profile $U$, (b) Temperature profile $\Theta$ and (c) Velocity profile $W$ for $D_{\rho}=0.0,100.0$ while $\operatorname{Pr}=0.7, \gamma=0.45, \alpha_{d}=1.0, \lambda=0.1$ and $X=1.54$. 

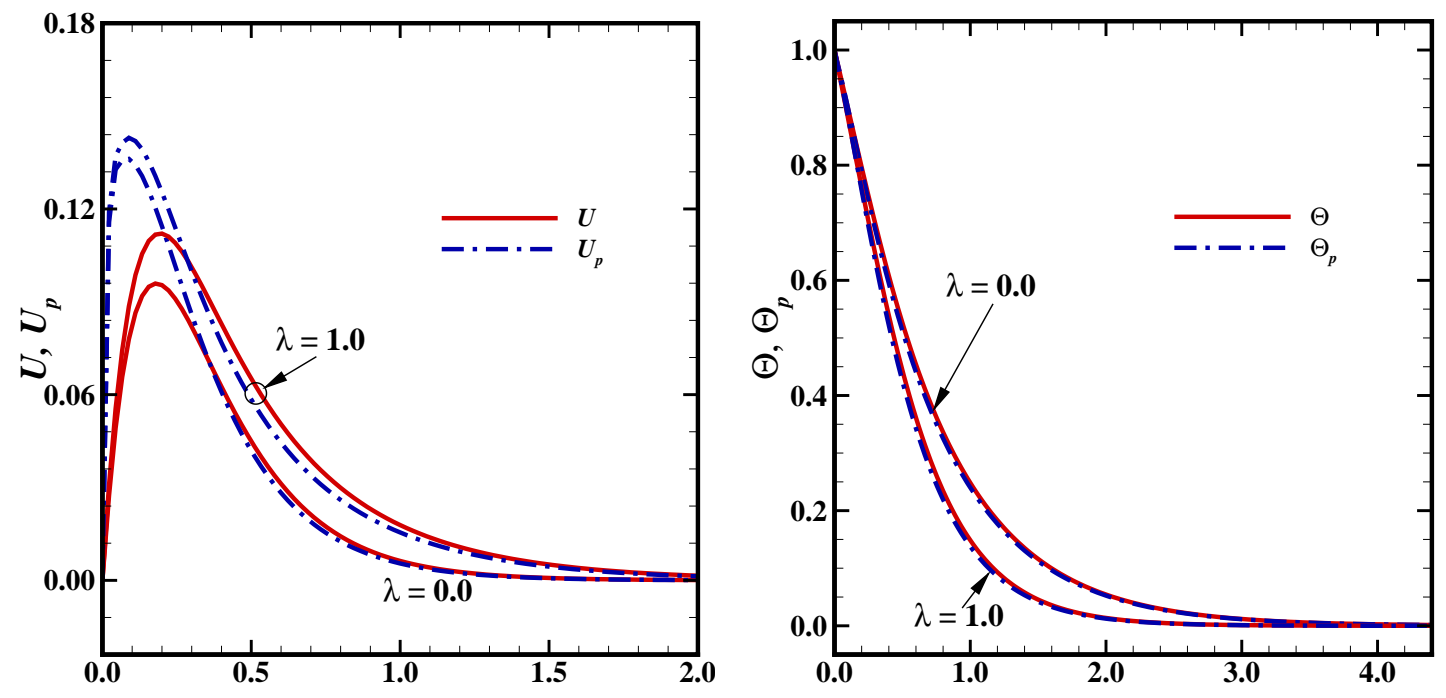

(a)

Y

(b)

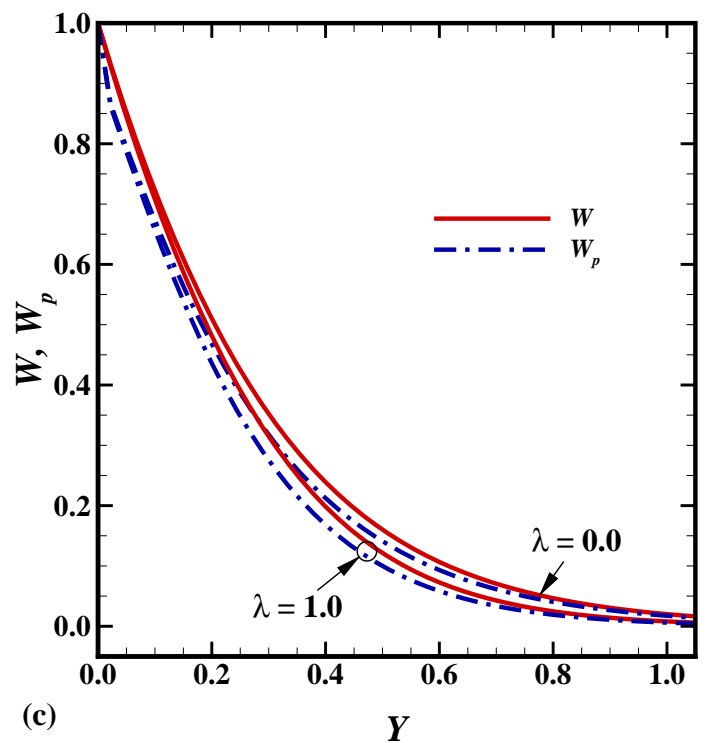

Fig. 7 (a) Velocity profile $U$, (b) Temperature profile $\Theta$ and (c) Velocity profile $W$ for $\lambda=0.0,1.0$ while $\mathbf{P r}=0.7, \gamma=0.45, \alpha_{d}=1.0, D_{\rho}=100.0$ and $X=1.54$. 